\title{
Anti-Inflammatory Effects of Protein Kinase Inhibitor Pyrrol Derivate
}

\author{
Halyna M. Kuznietsova, Maryna S. Yena, Iryna P. Kotlyar, \\ Olexandr V. Ogloblya, and Volodymyr K. Rybalchenko
}

Institute of Biology, Taras Shevchenko National University, Volodymyrska 64/13, Kyiv 01601, Ukraine

Correspondence should be addressed to Halyna M. Kuznietsova; biophyz@gmail.com

Received 28 July 2016; Revised 22 November 2016; Accepted 30 November 2016

Academic Editor: João Batista T. Da Rocha

Copyright (c) 2016 Halyna M. Kuznietsova et al. This is an open access article distributed under the Creative Commons Attribution License, which permits unrestricted use, distribution, and reproduction in any medium, provided the original work is properly cited.

In our previous studies we showed antitumor and anti-inflammatory activities of protein kinases inhibitor pyrrol derivate 1-(4Cl-benzyl)-3-Cl-4-(CF3-fenylamino)-1H-pyrrol-2,5-dione (MI-1) on rat colon cancer model. Therefore anti-inflammatory effect of MI-1 on rat acetic acid induced ulcerative colitis (UC) model was aimed to be discovered. The anti-inflammatory effects of MI-1 $(2.7 \mathrm{mg} / \mathrm{kg}$ daily) compared to reference drug Prednisolone $(0.7 \mathrm{mg} / \mathrm{kg}$ daily $)$ after 14-day usage were evaluated on macro- and light microscopy levels and expressed in 21-grade scale. Redox status of bowel mucosa was also estimated. It was shown that in UC group the grade of total injury (GTI) was equal to $9.6\left(\mathrm{GTI}_{\text {control }}=0\right)$. Increase of malonic dialdehyde $(\mathrm{MDA})$ by $89 \%$ and protein carbonyl groups (PCG) by $60 \%$ and decrease of superoxide dismutase (SOD) by $40 \%$ were also observed. Prednisolone decreased GTI to 3 and leveled SOD activity, but MDA and PCG remained higher than control ones by $52 \%$ and $42 \%$, respectively. MI- 1 restored colon mucosa integrity and decreased mucosa inflammation down to GTI $=0.5$ and leveled PCG and SOD. Thus, MI-1 possessed anti-inflammatory properties, which were more expressed that Prednisolone ones, as well as normalized mucosa redox balance, and so has a prospect for correction of inflammatory processes.

\section{Introduction}

Diseases of the digestive system occupied the second place among all human pathologies after respiratory ones. Inflammatory bowel disease (IBD), which includes ulcerative colitis (UC), is one of the most difficult diseases of the gut for clinical outcome, prevalence, and prognosis. The prevalence of UC in different European countries ranges from 50 to 200 cases per 100000 population [1]. Moreover, chronic UC has been shown to increase the risk of colorectal cancer up to 8 times [2].

Chronic UC is characterized by erosions and ulcers development in the colon mucosa (in the acute stage), mucosa inflammation, and surface epithelium injury [3]. Very often colon mucosa regeneration is impaired, lipid peroxidation is escalated, and antioxidant system functionality is oppressed [4]. Chronic form of UC in most cases follows the malignant tumor development [5].
The basic aims of chronic UC treatment are to dilute the inflammation and to inhibit the abnormal cell proliferation due to enhanced mucosa regeneration. The primary drugs used in UC traditionally include anti-inflammatory drugs (including ones based on 5-aminosalicylic acid), steroids, and immunosuppressive drugs [6]. However, traditional medications have side effects such as erosion, hemorrhage, and dysfunction of the gut, hepatitis, and pancreatitis, depression of hematopoiesis, and increased risk of infections. Moreover, the risk and severity of them increase with prolonged drugs administration. Furthermore, in some cases the traditional treatment is insufficient to achieve and maintain the remission. So, the need for more potent and more reliable medications is clear.

Protein kinase inhibitor pyrrol derivate 1-(4-Cl-benzyl)3-Cl-4-( $\mathrm{CF}_{3}$-fenylamino)-1H-pyrrol-2,5-dione (MI-1) (Figure 1) has been synthesized at Chemistry Department of Taras Shevchenko National University of Kyiv. MI-1 could 


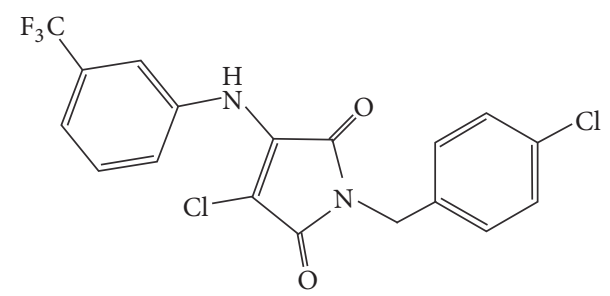

Figure 1: 1-(4-Cl-benzyl)-3-Cl-4-( $\mathrm{CF}_{3}$-fenylamino)-1H-pyrrol-2,5dione (MI-1): structural formula.

inhibit such protein kinases as Yes, Src(h), ZAP70, Syk(h), PDK1, EGFR, IGF-1R, VEGFR, and so forth as evidenced by $[7,8]$. MI-1 reveals antiproliferative and proapoptotic properties to malignant cells without significant effects on the proliferation and survival of normal ones [7, 9]. Antitumor activity of the agent was also demonstrated on rat model of chemically induced colon cancer [10]. Moreover, MI-1 is relatively safe for the digestive, reproductive, and excretory systems under chronic exposure, as evidenced by [11-13]. Furthermore, when administered to rats experiencing colon cancer, MI-1 attenuates inflammation of mucosa, adjacent to colorectal tumors $[10,11]$. MI-1 also reveals antioxidant properties on $\mathrm{CoCl}_{2}$-induced oxidative stress model [14].

As MI-1 possesses antitumor and anti-inflammatory activities against experimental colon cancer, as well as antioxidant ones against $\mathrm{CoCl}_{2}$-induced oxidative stress; the antiinflammatory and antioxidant effects of MI-1 on rat UC model were aimed at being discovered. Prednisolone, which is commonly used for moderate UC treatment [6], was chosen as reference drug.

\section{Materials and Methods}

2.1. Animals. All experimental procedures executed with animals were in compliance with international principles of the European Convention for the protection of vertebrate animals used for experimental and other scientific purposes (European convention, Strasburg, 1986), article 26 of the Law of Ukraine "On protection of animals from cruelty" (No 3447-IV, 21.02.2006), and all norms of bioethics and biosafety.

40 male Wistar rats weighing 200-220g (10 weeks old) were obtained from Central Animal House of Taras Shevchenko National University (Kyiv, Ukraine). Five animals were housed per plastic cage on softwood chip bedding and were maintained under constant conditions (12 hr light/ dark cycle, $60 \%$ humidity at $20-22^{\circ} \mathrm{C}$ ) and fed on standard diet and tap water ad libitum.

2.2. Chemicals. Ice acetic acid (99.8\%, VWR Prolabo, UK) was dissolved immediately before use in distilled water to obtain 4\% solution. To induce UC, animals were treated with $1 \mathrm{~mL} 4 \%$ acetic acid solution per rectum weekly for 2 weeks. MI-1 ( $\geq 98 \%$, Taras Shevchenko National University of Kyiv) was dissolved in vegetable oil. Animals were treated by MI-1 at the dose of $2.7 \mathrm{mg} / \mathrm{kg}$ of body weight per os daily. The common anti-inflammatory therapeutic Prednisolone (solution for injections, BioPharma, Ukraine) was used for referencing. Prednisolone was injected intraperitoneally daily at the dose of $0.7 \mathrm{mg} / \mathrm{kg}$ of body weight (according to clinical recommendations for moderate UC treatments [6]) in saline.

2.3. Experimental Design. Chronic UC was induced as described [15]: animals were induced by 2 rectal applications of $4 \%$ acetic acid $(1 \mathrm{~mL})$ weekly. After administration, the rats were held upside down for approximately 30 seconds to prevent immediate leakage of the agent from the anus. $15 \mathrm{~min}$ before acetic acid applications animals received 3$4 \mathrm{~mL}$ of saline intracolonically followed by light belly massage to empty the bowel. MI-1 and Prednisolone applications were started simultaneously with the administration of acetic acid and continued for 2 weeks. Control animals received the appropriate vehicles: saline or vegetable oil.

The rats were divided into 4 groups (10 rats each): (1) vehicle-treated control, (2) UC, the animals were treated with acetic acid, (3) UC + Prednisolone, the animals were treated with acetic acid and with Prednisolone, and (4) UC + MI-1, the animals were treated with acetic acid and with MI-1.

2.4. Macroscopic Assay. 1 day after the last treatment the rats were sacrificed by carbon dioxide asphyxia, the abdomen was opened, and the entire gastrointestinal tract was removed. The internal bowel surface was visually inspected, the mucosal lesions were scored macroscopically using the following scale: 0 , no damage; 1 , localized hyperemia and no ulcers; 2, ulceration without hyperemia or bowel wall thickening; 3 , ulceration with inflammation at one site; 4 , two or more sites of ulceration/inflammation; 5, major sites of damage extending more than $1 \mathrm{~cm}$ along the length of colon; $6-10$, damage extending more than $2 \mathrm{~cm}$ along the length of colon, where the score is increased by one for each additional $1 \mathrm{~cm}$ damage [16].

2.5. Histological Assay. The $1 \mathrm{~cm}$ colons (from 6 to $5 \mathrm{~cm}$ proximal to the anus) (distal colon) were fixed for 14 days in neutral saline containing $10 \%$ formalin. Then, they were embedded into paraffin and sliced into $5 \mu \mathrm{m}$ sections, which were stained with hematoxylin-eosin-orange [17] and examined under the light microscope (Olympus BX-41, Olympus Europe $\mathrm{GmbH}$, Japan). The mucosal lesions were scored using 11-grade scale, where total grade was calculated as a sum of grades for bowel mucosa integrity disorders (0-3 grades), leucocytic infiltration ( $0-3$ grades), muscle layer thickening (0-3 grades), crypt abscesses forming (0-1 grade), and goblet cells decrease (0-1 grades) [18]. The grade of total injury (GTI) was also calculated as a sum of grades for mucosa macro- and microscopic lesions.

Mucosa thickness, colonocytes height, and their nuclei cross-sectional area, as well as goblet cells one, were measured from the microphotographs with magnification 400x, using WCIF ImageJ software. The mitotic index (MI) was calculated as the sum of epithelial cells in any phase of mitosis divided on the total sum of epithelial cells. Goblet cells index (GI) and crypt fission index (CFI) were calculated as the sum of goblet cells/fissile crypts divided on the total sum of epithelial cells/crypts. 
2.6. Biochemical Assays. Bowel internal surfaces were washed with saline followed by PBS containing $1 \mathrm{mM}$ EDTA and 0.4 mM PMSF (serine proteases inhibitor) having $\mathrm{pH}$ 7.0. Then mucosa was scraped away and rapidly frozen at $-70^{\circ} \mathrm{C}$. After being thawed, the samples were gently homogenized in PBS containing $1 \mathrm{mM}$ EDTA and $0.4 \mathrm{mM}$ PMSF and centrifuged at $10000 \mathrm{~g}$ for $15 \mathrm{~min}$, and supernatants were collected and used for analysis. Total protein was estimated quantitatively as described by Lowry et al. [19]. Malonic dialdehyde (MDA), protein carbonyl groups (PCG), intracellular superoxide dismutase (SOD), and catalase (CAT) activities as indicators of colon mucosa redox status were measured spectrophotometrically.

MDA was estimated quantitatively by reaction with thiobarbituric acid as described by [20]. Chromogen absorbance was determined at $\lambda=532 \mathrm{~nm}$ at room temperature against blank reference. The extent of lipid peroxidation was expressed as MDA (nmol per mg protein) using a molar extinction coefficient for MDA of $1.56 \times 10^{5} \mathrm{M}^{-1} \mathrm{~cm}^{-1}$. The level of oxidized proteins (protein carbonyl groups) was estimated quantitatively by oxidized amino acid residues $+2,4$ dinitrophenylhydrazine method as described by [21]. Chromogen absorbance was determined at $\lambda=370 \mathrm{~nm}$ at room temperature against blank reference. The extent of oxidized proteins was expressed as 2,4-dinitrophenylhydrazone (nmol per mg protein) using a molar extinction coefficient for 2,4dinitrophenylhydrazone of $2.2 \cdot 10^{4} \mathrm{M}^{-1} \mathrm{~cm}^{-1}$. Superoxide dismutase (SOD) activity was quantitatively assessed by nitro blue tetrazolium + riboflavin method as described by [20]. Reaction of $\mathrm{O}_{2}{ }^{-}$generation (and nitro blue tetrazolium recovery to formazan) was initiated by bright sunshine for $10 \mathrm{~min}$; formazan absorbance was determined at $\lambda=540 \mathrm{~nm}$ at room temperature against blank reference. One unit of SOD activity was considered as $1 \%$ inhibition of formazan occurrence. CAT activity was quantitatively assessed by $\mathrm{H}_{2} \mathrm{O}_{2}$ + molybdenum salt method as described by [22]. Reaction was initiated by adding of $0.03 \% \mathrm{H}_{2} \mathrm{O}_{2}$ solution to analyzed supernatant and stopped after $10 \mathrm{~min}$ by adding of $4 \%$ ammonium molybdate solution. Chromogen absorbance was determined at $\lambda=410 \mathrm{~nm}$ at room temperature against blank reference. CAT activity was expressed as $\mathrm{H}_{2} \mathrm{O}_{2}$ splitting ( $\mathrm{mmol} / \mathrm{mg}$ protein per $\mathrm{min})$ using a molar extinction coefficient for $\mathrm{H}_{2} \mathrm{O}_{2}$ of $22.2 \mathrm{M}^{-1} \mathrm{~cm}^{-1}$.

2.7. Statistical Analysis. The statistical significance of differences was determined by one-way analysis of variance (ANOVA) with the Bonferroni post hoc test. A value of $p \leq$ 0.05 was considered significant.

\section{Results}

3.1. Ulcerative Colitis Group. Bowel wall thickening, irritation and cornification, adhesions between bowel loops, colonic clefts, and small ulcers on internal bowel surface were observed after visual inspection under UC condition (Figure 2(b)). Edema, epithelium desquamation, fibrinoid, and necrotic layers at areas up to $2 \mathrm{~cm}$ along the bowel and inflammatory features manifested by submucosa lymphoid and histiocytic infiltration and extravasation were detected at light microscopy assay (Figure 3(b)). GTI was equal to 9.6 $\left(\mathrm{GTI}_{\text {control }}=0\right)$ (Figure 4). Mucosa morphometrial parameters in this group were similar to control ones (Table 1), so we concluded [23] the development of chronic UC without atrophy.

Increase of MDA (by 89\%) and PCG (by 60\%) (Figures 7 and 8 ) suggested the increase of lipid and protein oxidation and oxidative stress development $[24,25]$. Decrease of SOD activity (by 40\%) (Figure 6) in bowel mucosa suggested the excess of $\mathrm{H}_{2} \mathrm{O}_{2}$ or reactive oxygen species [26] and could provoke further escalation of lipid and protein peroxidation and redox imbalance. Also CAT activity tended to decrease compared to control one (Figure 6). These observations are typical for inflammatory processes [25] and confirm the UC development.

3.2. UC + Prednisolone Group. In this experimental group no bowel lesions, except light edema, were observed under visual inspection (Figure 2(c)), as well as no ulcers, edema, and epithelium desquamation at light microscopy analysis. Nevertheless, some inflammatory features (submucosa lymphoid and histiocytic infiltration) persisted (Figure 3(c)); thus GTI was equal to 3 (Figure 4). Mucosa morphometrial parameters in this group were similar to control ones (Table 1), but GI was less than UC one by $20 \%$, suggesting some inhibition of mucous formation and/or secretion.

SOD activity was leveled by Prednisolone (Figure 5), but MDA and PCG remained higher than control ones by $51 \%$ and $42 \%$, respectively, despite their decrease (by $20 \%$ and $11 \%$, resp.) compared with UC group (Figures 7 and 8). These findings suggested the attenuation of inflammation and oxidative stress level by Prednisolone, as well as partial restore of mucosa integrity.

3.3. UC + MI-1 Group. No bowel lesions after visual inspection (Figure 2(d)) and no ulcers, edema, and epithelium desquamation at microscopic level were observed at this group. Moreover, mucosa inflammation decreased down to local foci of lymphocytes and histiocytes (Figure 3(d)); thus GTI was equal to 0.5 (Figure 4). Mucosa morphometrial parameters in this group were similar to control ones (Table 1), but colonocytes' nuclei area exceeded the UC one by $23 \%$, suggesting the activation of colonocytes' functional state. Also CFI decreased by $54 \%$ compared to UC and tended to decrease by $44 \%$ compared to control ones.

MI-1 also leveled PCG and SOD (Figures 5 and 8), but MDA remained higher than control one by $43 \%$, despite its decrease (by 24\%) compared with UC group (Figure 7). So MI-1 more effectively restored colon mucosa integrity and attenuated inflammation and oxidative stress level than Prednisolone did.

\section{Discussion}

The mechanisms of anti-inflammatory action of glucocorticoids are well established. Thus, glucocorticoids are capable of suppressing the inflammatory process through altering the expression of corticosteroid-responsive genes. Glucocorticoid-specific receptors in the cell cytoplasm bind with 


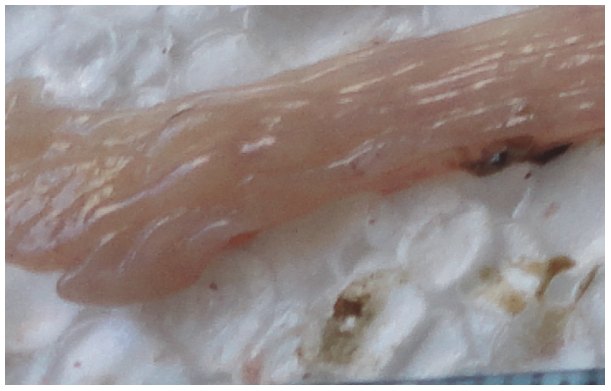

(a)

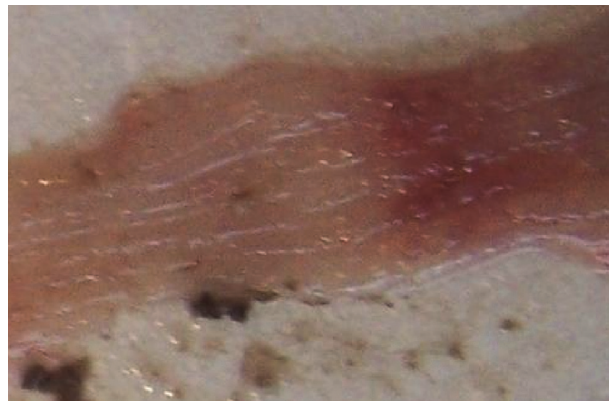

(c)

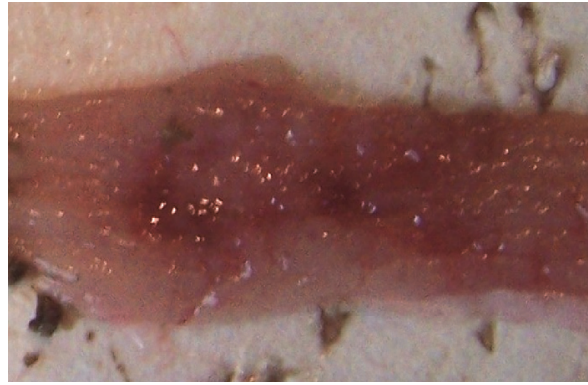

(b)

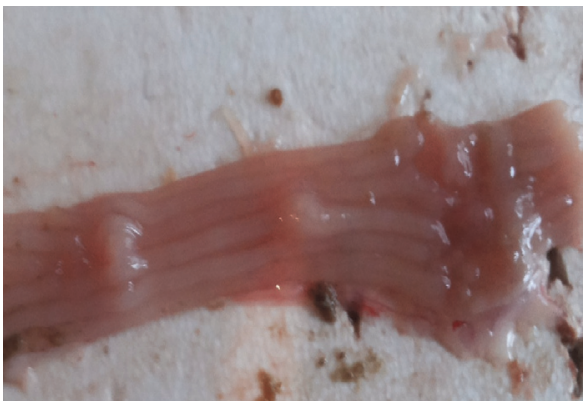

(d)

FIGURE 2: Macrophotographs of bowel internal sides for rats experienced UC and treated with Prednisolone and MI-1. (a) Control group, (b) UC group, (c) UC + Prednisolone group, and (d) UC + MI-1 group.

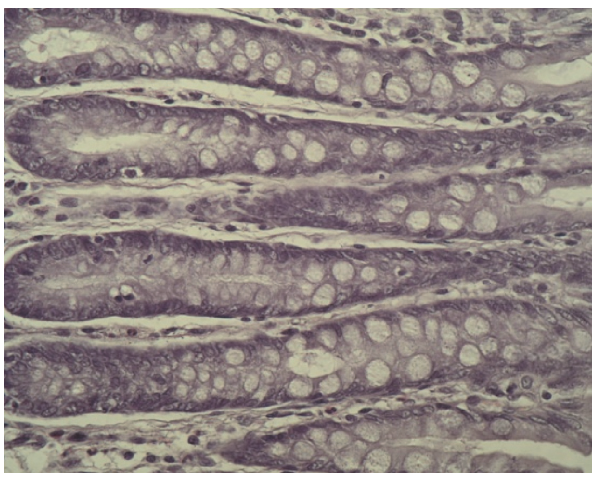

(a)

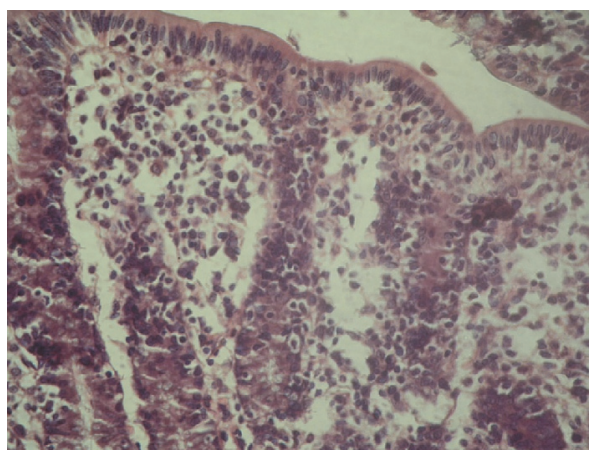

(c)

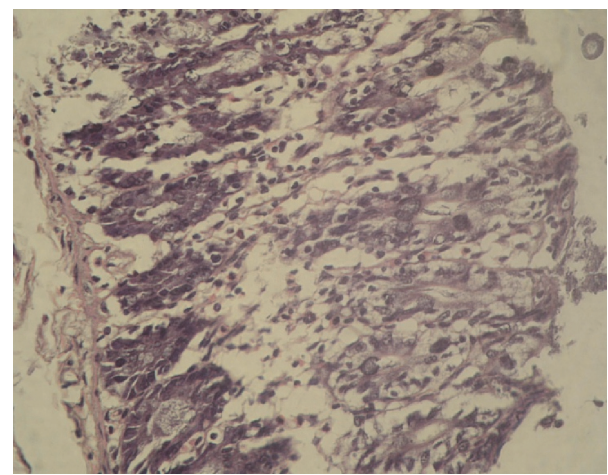

(b)

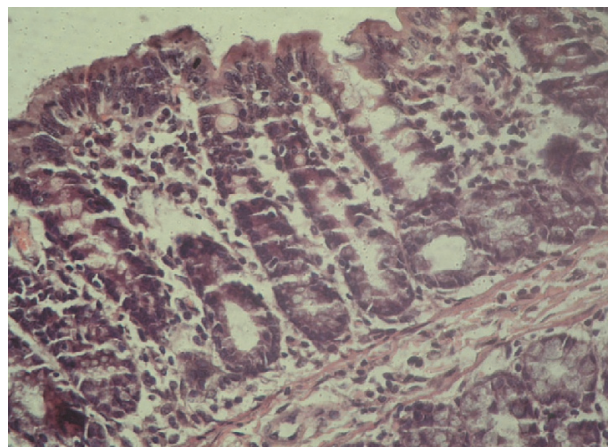

(d)

FIGURE 3: Microphotographs of upper colons for rats experiencing UC and treated with Prednisolone and MI-1. Hematoxylin-eosin orange staining, $\times 400$. (a) Control group, (b) UC group, (c) UC + Prednisolone group, and (d) UC + MI-1 group. 
TABLE 1: Morphometric parameters of colon mucosa for rats experiencing UC and treated with Prednisolone and MI-1. Mean \pm SD, $n=10^{*}$.

\begin{tabular}{|c|c|c|c|c|}
\hline & Control & UC & UC + Prednisolone & $\mathrm{UC}+\mathrm{MI}-1$ \\
\hline Mucosa thickness, $\mu \mathrm{m}$ & $588.5 \pm 93.8$ & $648.7 \pm 103.6$ & $651.5 \pm 129.4$ & $667.5 \pm 133.6$ \\
\hline Colonocytes height, $\mu \mathrm{m}$ & $17.2 \pm 3.6$ & $17.4 \pm 2.6$ & $16.4 \pm 2.4$ & $16.0 \pm 2.2$ \\
\hline $\begin{array}{l}\text { Colonocytes nuclei } \\
\text { cross-sectional area, } \mu \mathrm{m}^{2}\end{array}$ & $22.7 \pm 4.8$ & $22.0 \pm 4.2$ & $23.9 \pm 1.6$ & $\begin{array}{l}27.0 \pm 4.4 \\
(p=0.04)\end{array}$ \\
\hline $\begin{array}{l}\text { Goblet cells cross-sectional } \\
\text { area, } \mu \mathrm{m}^{2}\end{array}$ & $82.1 \pm 10.2$ & $69.4 \pm 18.0$ & $65.2 \pm 14.0$ & $71.9 \pm 8.4$ \\
\hline GI, \% & $26.7 \pm 3.2$ & $28.8 \pm 1.4$ & $\begin{array}{l}23.0 \pm 3.0 \\
(p=0.008)\end{array}$ & $27.5 \pm 3.8$ \\
\hline MI, \% & $5.2 \pm 0.8$ & $5.0 \pm 1.4$ & $4.6 \pm 1.2$ & $5.1 \pm 0.6$ \\
\hline CFI, \% & $5.7 \pm 2.6$ & $7.0 \pm 1.2$ & $6.4 \pm 1.4$ & $\begin{array}{c}3.2 \pm 1.0 \\
(p=0.007)\end{array}$ \\
\hline
\end{tabular}

${ }^{*} p$ values indicated in comparison with UC group.



FIGURE 4: Bowel injury for rats experiencing UC and treated with Prednisolone and MI-1. ${ }^{*} p \leq 0.05$ compared with control group; ${ }^{*} p \leq 0.05$ compared with UC group.

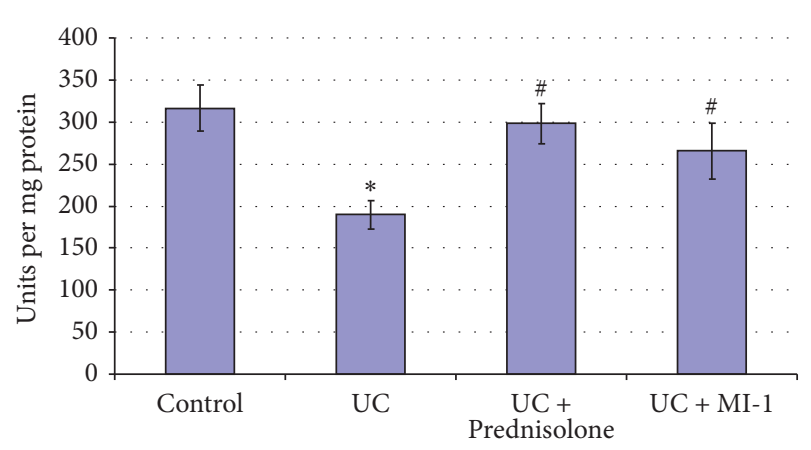

FIGURE 5: Bowel mucosa SOD activity for rats experiencing UC and treated with Prednisolone and MI-1. ${ }^{*} p \leq 0.05$ compared with control group; ${ }^{\#} p \leq 0.05$ compared with UC group.

steroid ligands to form hormone-receptor complexes that eventually translocate to the cell nucleus. There, these complexes bind to specific DNA sequences and alter their expression. The complexes may induce the transcription of mRNA, leading to synthesis of new proteins. Such proteins include lipocortin, a protein known to inhibit phospholipase A2 and thereby block the synthesis of prostaglandins and leukotrienes from arachidonic acid. Glucocorticoids also inhibit the production of other mediators, including

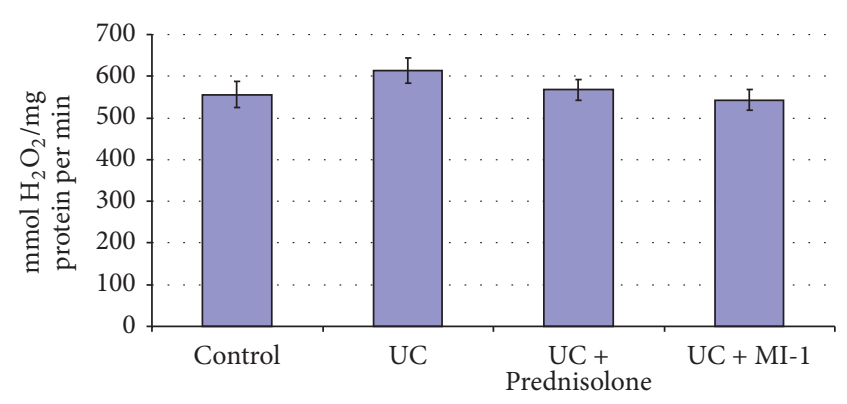

FIgURE 6: Bowel mucosa CAT activity for rats experiencing UC and treated with Prednisolone and MI-1.

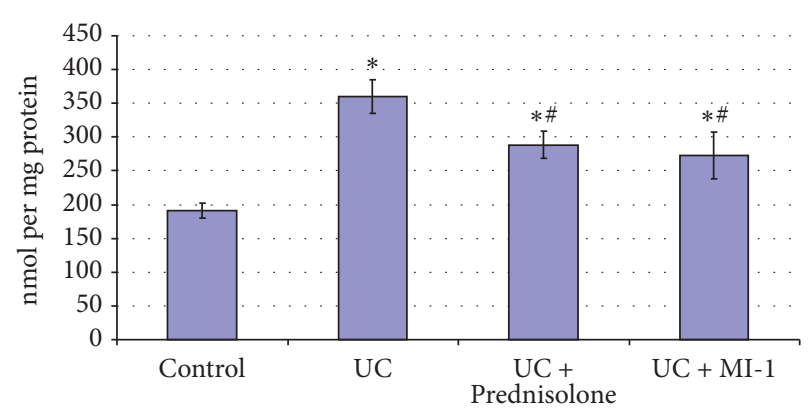

FIgURE 7: Bowel mucosa MDA concentrations for rats experiencing UC and treated with Prednisolone and MI-1. ${ }^{*} p \leq 0.05$ compared with control group; ${ }^{\#} p \leq 0.05$ compared with UC group.

arachidonic acid metabolites such as those produced via cyclooxygenase (COX) activation (both COX-1 and COX2), cytokines, interleukins, adhesion molecules, and enzymes such as collagenase. Hormone-receptor complex also has the ability to repress the activity of NF- $\kappa \mathrm{B}$, one of the main regulators of inflammatory response, directly $[27,28]$.

The possible mechanisms of action of tyrosine kinase inhibitors are not so clear. MI-1 was synthesized as protein kinases inhibitor [8]. It was tested on 32 protein kinases for determination of inhibitory profile and has been shown to inhibit EGF-R, FGF-R1, IGF1-R, INS-R, VEGF-R1, VEGF-R2, VEGF-R3, Syk, ZAP70, PDK1, Yes, and Src(h) [7]. It is known 


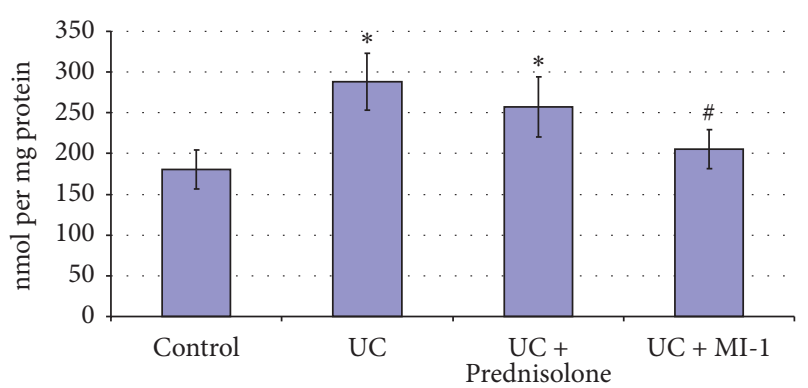

FIgUre 8: Bowel mucosa PCG concentrations for rats experiencing UC and treated with Prednisolone and MI-1. ${ }^{*} p \leq 0.05$ compared with control group; ${ }^{\#} p \leq 0.05$ compared with UC group.

that EGF-R, VEGFR, IGF1R, Src, and PDK1 are overexpressed in human colorectal tumors [29, 30], so the mechanism of MI-1 antitumour action could consist on inhibition of these kinases in tumor tissue. But it is also known that some of these receptors and proteins are involved in inflammatory process. Thus, EGFR and IGF1R, which are the targets of MI1 , act as the major upstream activators of PI3K/Akt pathway. Moreover, PDK1, another target of MI-1, is the key effector of this pathway. Activation of PI3K/Akt pathway leads to activation of NF- $\kappa \mathrm{B}$ [31], which regulates host inflammatory and immune responses, as well as cellular growth. Activation of NF- $\kappa \mathrm{B}$ pathway is involved in the pathogenesis of chronic inflammatory diseases, such as asthma, rheumatoid arthritis, and IBD. Inhibition of NF- $\kappa \mathrm{B}$ pathway by glucocorticoids and nonsteroidal anti-inflammatory drugs is associated with anti-inflammatory properties of these medications [32]. Thus, anti-inflammatory properties of EGFR-, IGF1R-, and PDK1inhibitors could be explained by inhibition of PI3K/Akt/NF$\kappa \mathrm{B}$ signaling pathway [33].

Recent publications suggest that altered angiogenesis may be a critical component of IBD pathogenesis. Angiogenesis in the area of chronic inflammation, such as that observed in IBD, is abnormal and characterized by distorted vasculature, increased permeability, and thrombogenic potential. Moreover, abnormal angiogenesis may facilitate migration of inflammatory cells to the site of inflammation, leading to the perpetuation of chronic inflammation. Indeed, angiogenesis is intrinsic to chronic inflammation and is associated with structural changes, including activation and proliferation of endothelial cells and capillary and venule remodeling, all of which result in expansion of the tissue microvascular bed [34]. A potential functional consequence of this expansion is the promotion of inflammation through increase of inflammatory cells influx and nutrient supply to the metabolically active immune process and the local production of cytokines, chemokines, and matrix metalloproteinases by activated endothelium [35]. Thus, angiogenesis and inflammation become chronically codependent processes. It has now been clearly established that the microvascular changes associated with angiogenesis are key contributors to the tissue injury and remodeling process that accompanies chronic inflammation. VEGF is the predominant regulator of angiogenesis through the initiation of vessel growth, the inhibition of endothelial cell apoptosis, and the incorporation of hematopoietic and endothelial progenitor cells into the developing vasculature. Because growing evidence supports a role for angiogenesis in chronic inflammation [36], the inhibition of VEGF signaling has also been proposed as a promising therapeutic strategy in this disorder. In fact, it was shown that the inhibition of VEGF signaling in dextran sodium sulfate-induced colitis reduces inflammation, whereas the overexpression of VEGF leads to an augmentation of intestinal inflammation [37]. It was also evidenced [38] that in inflamed tissue VEGF is highly expressed not only in endothelial cells, but also in leukocytes and epithelial cells. Treatment with anti-VEGF antibody markedly improves the clinical and morphologic features of UC in part by reducing excessive vascular permeability and decreasing inflammatory cells infiltration. Therefore, antiinflammatory effects of MI-1 could be mediated by VEGFR pathway blockade.

Syk is a key protein of the immunoreceptor signaling pathway in immune and inflammatory responses. Syk has been proposed to play a critical role in psoriasis, atherosclerosis, and IBD. The data [39] showed that Syk expression increases in experimental colitis, and fostamatinib, a Syk inhibitor, affects inflammatory cells and proinflammatory cytokines and provides histological and morphological healing of colon mucosa. MI-1 could inhibit Syk in vitro, so its anti-inflammatory effects could be realized through signaling pathways which involve Syk.

Oxidative stress, among the immune-regulatory factors, has been proposed as one of the major mechanisms involved in the pathophysiology of IBD. The predominant source of reactive oxygen species (ROS) in the gut mucosa is the NADPH oxidases (NOX). NOX 1 is highly expressed in colon epithelial cells, where it generates ROS to interact with normal and pathogenic bacteria. Excessive reactive ROS production is associated with damage to the intestinal mucosa at IBD. Moreover, chronic inflammation in IBD is characterized by massive leukocyte infiltration of the gut. On activation, these cells produce not only a wide spectrum of proinflammatory cytokines but also an excessive amount of ROS, which markedly alters the redox equilibrium within the gut mucosa, and maintain inflammation by inducing redoxsensitive signaling pathways and transcription factors. Recent developments point to the possibility of treating the oxidative stress associated with IBD by using chemical compounds with antioxidant potential [4]. In our study we showed that MI-1 decreases the formation of lipid and protein peroxides and normalizes the attenuated SOD activity and so acts like an antioxidant. In our previous studies antioxidant properties of MI-1 were also observed [40]. Thereby we propose that MI1 could impact tissue redox equilibrium through inhibition of proinflammatory signaling pathways (e.g., PI3K/Akt/NF$\kappa \mathrm{B})$.

It was shown that activation of EGFR is associated with ROS production, which transiently inactivates protein tyrosine phosphatases to enhance or prolong EGFR activation [6]. Moreover, abnormalities in EGFR activation in the context of chronic diseases such as cancer or IBD may not only simply be due to altered expression of EGFR or its ligands but also be related to altered expression or activation of 
NOX enzymes that regulate this signaling pathway [41]. Also exogenous ROS stimulates the induction of VEGF expression in endothelial cells, smooth muscle cells, and macrophages. At the same time, VEGF further stimulates ROS production through the activation of NOX in endothelial cells. In several pathologies, exemplified by diabetic retinopathy and injured arteries, ROS-mediated angiogenesis is strongly associated with VEGF expression [42]. So inhibition of EGFR and VEGFR could be associated with decreased ROS production. Therefore we propose that MI-1 could inhibit ROS production and normalize impaired redox balance through inhibition of EGFR and VEGFR.

Indeed, protein kinases are strongly involved in pathogenesis of IBD and thereby could be the targets of anti-IBD therapy. For example, tofacitinib which is a small molecular inhibitor of the Janus kinase is currently approved for the treatment of rheumatoid arthritis and recently has been shown to be effective in the treatment of UC [43]. So the therapeutic potential of targeted protein kinase inhibitors including pyrrol derivate MI-1 is extended.

\section{Conclusions}

MI-1 possessed anti-inflammatory properties more expressed than Prednisolone ones, as well as normalized mucosa redox equilibrium. Since anti-inflammatory effects of glucocorticoids are realized through inhibition of phospholipase A2 and COX-2, whereas effects of MI-1 through inflammatory and ROS signaling pathways are multiple, probably determining more expressed and complex impact of MI-1 on inflammatory process. Thus MI-1 has a prospect for correction of inflammatory process through multiple mechanisms of action.
Abbreviation List
IBD: Inflammatory bowel disease
UC: Ulcerative colitis
MI-1: 1-(4-Cl-benzyl)-3-Cl-4-(CF3-fenylamino)- $1 \mathrm{H}$-pyrrol-2,5-dione
Yes: Yamaguchi sarcoma viral oncogene homolog 1
Src(h): Rous sarcoma oncogene cellular homolog
ZAP70: Zeta-chain-associated protein kinase 70
Syk(h): Spleen tyrosine kinase
PDK1: 3-Phosphoinositide-dependent kinase 1
EGFR: Endothelial growth factor receptor
IGF1R: Insulin-like growth factor-1 receptor
VEGFR: Vasoendothelial growth factor receptor
GTI: Grade of total injury
MI: $\quad$ Mitotic index
GI: $\quad$ Goblet cells index
CFI: $\quad$ Crypt fission index
PBS: $\quad$ Phosphate-buffered saline
EDTA: Ethylenediaminetetraacetic acid
PMSF: Phenylmethylsulphonyl fluoride
MDA: Malonic dialdehyde
PCG: Protein carbonyl groups
SOD: Superoxide dismutase
CAT:
Catalase
COX: Cyclooxygenase
INS-R: Insulin receptor
PI3K/Akt: Phosphatidylinositol-3 kinase/protein kinase B
NF- $\kappa \mathrm{B}: \quad$ Nuclear factor $\kappa \mathrm{B}$
ROS: $\quad$ Reactive oxygen species
NOX: Nicotinamide adenine dinucleotide phosphate oxidase.

\section{Competing Interests}

The authors declare that there is no conflict of interests regarding the publication of this paper.

\section{Acknowledgments}

The research was carried out in the frame of the Fundamental research grant of Ministry of Education and Science of Ukraine "Investigation of the mechanisms of digestive system functions and elaboration of the methods for their correction" (no. 0106U005755).

\section{References}

[1] P. L. Lakatos, "Recent trends in the epidemiology of inflammatory bowel diseases: up or down?" World Journal of Gastroenterology, vol. 12, no. 38, pp. 6102-6108, 2006.

[2] P. L. Lakatos and L. Lakatos, "Risk for colorectal cancer in ulcerative colitis: changes, causes and management strategies," World Journal of Gastroenterology, vol. 14, no. 25, pp. 3937-3947, 2008.

[3] J. D. Feuerstein and A. S. Cheifetz, "Ulcerative colitis: epidemiology, diagnosis, and management," Mayo Clinic Proceedings, vol. 89, no. 11, pp. 1553-1563, 2014.

[4] I. Balmus, A. Ciobica, A. Trifan, and C. Stanciu, "The implications of oxidative stress and antioxidant therapies in Inflammatory Bowel Disease: clinical aspects and animal models," Saudi Journal of Gastroenterology, vol. 22, no. 1, pp. 3-17, 2016.

[5] T. A. Ullman and S. H. Itzkowitz, "Intestinal inflammation and cancer," Gastroenterology, vol. 140, no. 6, pp. 1807.e1-1816.e1, 2011.

[6] C.-T. Xu, S.-Y. Meng, and B.-R. Pan, "Drug therapy for ulcerative colitis," World Journal of Gastroenterology, vol. 10, no. 16, pp. 2311-2317, 2004.

[7] G. Dubinina, S. Golovach, V. Kozlovsky et al., "Antiproliferative action of the new derivatives of 1-(4-R-benzyl)-3-R1-4(R2-phenylamino)-1H-pyrrol-2,5-dione," Journal of Organ and Pharm Chem, vol. 5, no. 1, pp. 39-49, 2007.

[8] G. G. Dubinina, O. O. Chupryna, M. O. Platonov et al., "In silico design of protein kinase inhibitors: successes and failures," AntiCancer Agents in Medicinal Chemistry, vol. 7, no. 2, pp. 171-188, 2007.

[9] L. V. Garmanchuk, E. O. Denis, V. V. Nikulina et al., "MI1-derivative of maleimide inhibits cell cycle progression in tumor cells of epithelial origin," Biopolymers and Cell, vol. 29, no. 1, pp. 7074, 2013.

[10] O. Lynchak, G. Ostrovska, A. Burlaka et al., "State of colon mucosal under the effects of new protein-tyrosine kinases inhibitor maleimide derivate," Gut, vol. 59, supplement 3, article A133, 2010. 
[11] H. M. Kuznietsova, O. V. Lynchak, M. O. Danylov, I. P. Kotliar, and V. K. Rybal'chenko, "Effect of dihydropyrrol and maleimide derivatives on the state of the liver and colon in normal rats and those with colorectal carcinogenesis induced by dimethylhydrazine," Ukrainskǐ Biokhimicheskǐ Zhurnal, vol. 85, no. 3, pp. 74-84, 2013.

[12] I. V. Byelinska, O. V. Lynchak, T. V. Rybalchenko, S. V. Yablonska, O. M. Bahurynska, and V. K. Rybalchenko, "Morphofunctional parameters of blood cells of a rat with 1,2-dimethylhydrazine-induced colon carcinogenesis," Cytology and Genetics, vol. 49, no. 3, pp. 158-164, 2015.

[13] I. Kharchuk, N. Karpezo, G. Ostrovska et al., "The morphofunctional changes in the rat's testes under the influence of the new antineoplastic medicine derivative of maleimide," Modern Problems of Toxicology, vol. 1, pp. 61-65, 2008.

[14] S. V. Yablonska, O. M. Filinska, G. V. Ostrovska, and V. K. Rybalchenko, "Evaluation of hepatotoxisity of novel maleimide derivative with cytostatic activity and its influense on peroxidation process and antioxidant system in liver," The Ukrainian Biochemical Journal, vol. 81, no. 5, pp. 83-92, 2009.

[15] A. Dugani, B. Dakhil, and S. Treesh, "Protective effect of the methanolic extract of malva parviflora l. leaves on acetic acidinduced ulcerative colitis in rats," Saudi Journal of Gastroenterology, vol. 22, no. 3, p. 225, 2016.

[16] A. Ö. Şehirli, E. Tatlidede, M. Yüksel et al., "Protective effects of $\alpha$-lipoic acid against oxidative injury in TNBS-induced colitis," Erciyes Tip Dergisi, vol. 31, no. 1, pp. 015-026, 2009.

[17] J. Kiernan, Histological and Histochemical Methods: Theory and Practice, Cold Spring Harbor Laboratory Press, New York, NY, USA, 4th edition, 2008.

[18] C. B. Appleyard and J. L. Wallace, "Reactivation of hapteninduced colitis and its prevention by anti- inflammatory drugs," American Journal of Physiology, vol. 269, no. 1, pp. G119-G125, 1995.

[19] O. H. Lowry, N. J. Rosebrough, A. L. Farr, and R. J. Randall, "Protein measurement with the Folin phenol reagent," The Journal of Biological Chemistry, vol. 193, no. 1, pp. 265-275, 1951.

[20] V. Artyukhov and M. Nakvasina, Biological Membranes: Structural Organization, Functions, Modification by Physical and Chemical Agents, Voronezh University Press, Voronezh, Russia, 2000.

[21] R. L. Levine, D. Garland, C. N. Oliver et al., "Determination of carbonyl content in oxidatively modified proteins," Methods in Enzymology, vol. 186, pp. 464-478, 1990.

[22] L. Góth, "A simple method for determination of serum catalase activity and revision of reference range," Clinica Chimica Acta, vol. 196, no. 2-3, pp. 143-151, 1991.

[23] W. Finkbeiner and R. Davis, Autopsy Pathology: A Manual and Atlas, Churchill Livingstone, New York, NY, USA, 2003.

[24] G. Yu, I. Belenichev, E. Levitsky et al., “Toxicological outcomes of protein oxidative modification under different pathological states," Modern Problems of Toxicology, vol. 3, pp. 20-26, 2005.

[25] M. Mittal, M. R. Siddiqui, K. Tran, S. P. Reddy, and A. B. Malik, "Reactive oxygen species in inflammation and tissue injury," Antioxidants \& Redox Signaling, vol. 20, no. 7, pp. 1126-1167, 2014.

[26] E. Pigeolet, P. Corbisier, A. Houbion et al., "Glutathione peroxidase, superoxide dismutase, and catalase inactivation by peroxides and oxygen derived free radicals," Mechanisms of Ageing and Development, vol. 51, no. 3, pp. 283-297, 1990.

[27] R. Newton, "Molecular mechanisms of glucocorticoid action: what is important?” Thorax, vol. 55, no. 7, pp. 603-613, 2000.
[28] A. E. Coutinho and K. E. Chapman, "The anti-inflammatory and immunosuppressive effects of glucocorticoids, recent developments and mechanistic insights," Molecular and Cellular Endocrinology, vol. 335, no. 1, pp. 2-13, 2011.

[29] M. Takahashi and K. Wakabayashi, "Gene mutations and altered gene expression in azoxymethane-induced colon carcinogenesis in rodents," Cancer Science, vol. 95, no. 6, pp. 475-480, 2004.

[30] M. Kanneganti, M. Mino-Kenudson, and E. Mizoguchi, "Animal models of colitis-associated carcinogenesis," Journal of Biomedicine and Biotechnology, vol. 2011, Article ID 342637, 23 pages, 2011.

[31] I. Hers, E. E. Vincent, and J. M. Tavaré, "Akt signalling in health and disease," Cellular Signalling, vol. 23, no. 10, pp. 1515-1527, 2011.

[32] Y. Yamamoto and R. B. Gaynor, "Therapeutic potential of inhibition of the NF- $\kappa \mathrm{B}$ pathway in the treatment of inflammation and cancer," Journal of Clinical Investigation, vol. 107, no. 2, pp. 135-142, 2001.

[33] C. M. Blakely, E. Pazarentzos, V. Olivas et al., "NF- $\kappa$ B-Activating complex engaged in response to EGFR oncogene inhibition drives tumor cell survival and residual disease in lung cancer," Cell Reports, vol. 11, no. 1, pp. 98-110, 2015.

[34] P. Carmeliet, "Mechanisms of angiogenesis and arteriogenesis," Nature Medicine, vol. 6, no. 4, pp. 389-395, 2000.

[35] Z. Szekanecz and A. E. Koch, "Vascular endothelium and immune responses: implications for inflammation and angiogenesis," Rheumatic Disease Clinics of North America, vol. 30, no. 1, pp. 97-114, 2004.

[36] P. Carmeliet, "Angiogenesis in health and disease," Nature Medicine, vol. 9, no. 6, pp. 653-660, 2003.

[37] F. Scaldaferri, S. Vetrano, M. Sans et al., "VEGF-a links angiogenesis and inflammation in inflammatory bowel disease pathogenesis," Gastroenterology, vol. 136, no. 2, pp. 585-595, 2009.

[38] G. Tolstanova, T. Khomenko, X. Deng et al., "Neutralizing antiVascular Endothelial Growth Factor (VEGF) antibody reduces severity of experimental ulcerative colitis in rats: direct evidence for the pathogenic role of VEGF," Journal of Pharmacology and Experimental Therapeutics, vol. 328, no. 3, pp. 749-757, 2009.

[39] G. Can, S. Ayvaz, H. Can et al., "The Syk inhibitor fostamatinib decreases the severity of colonic mucosal damage in a rodent model of colitis," Journal of Crohn's and Colitis, vol. 9, no. 10, pp. 907-917, 2015.

[40] O. Filinska, S. Yablonska, S. Mandryk et al., "Effect of maleimide derivative on oxidative stress and glutathione antioxidant system in 1,2-dimethylhydrazine induced colon carcinogenesis in rat," Annales Universitatis Mariae Curie-Sklodowska, Sectio DDD: Pharmacia, vol. 23, no. 3, pp. 191-195, 2010.

[41] D. E. Heppner and A. van der Vliet, "Redox-dependent regulation of epidermal growth factor receptor signaling," Redox Bio$\log y$, vol. 8, pp. 24-27, 2016.

[42] Y.-W. Kim and T. V. Byzova, "Oxidative stress in angiogenesis and vascular disease," Blood, vol. 123, no. 5, pp. 625-631, 2014.

[43] J. Panés, C. Su, A. G. Bushmakin, J. C. Cappelleri, C. Mamolo, and P. Healey, "Randomized trial of tofacitinib in active ulcerative colitis: analysis of efficacy based on patient-reported outcomes," BMC Gastroenterology, vol. 15, no. 14, article 9, 2015. 




The Scientific World Journal
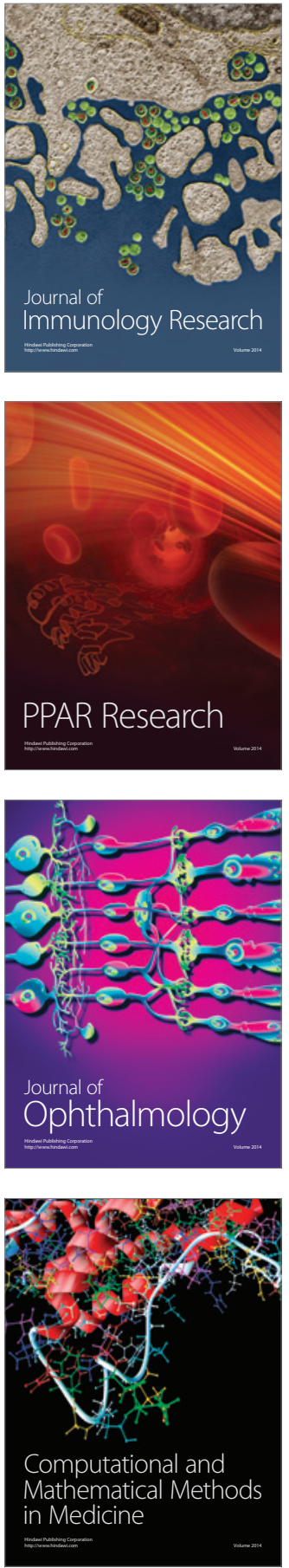

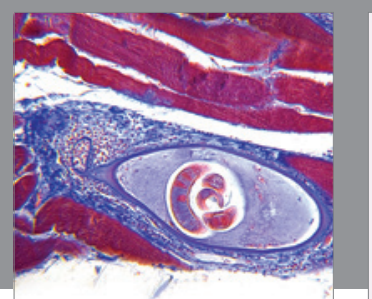

Gastroenterology Research and Practice

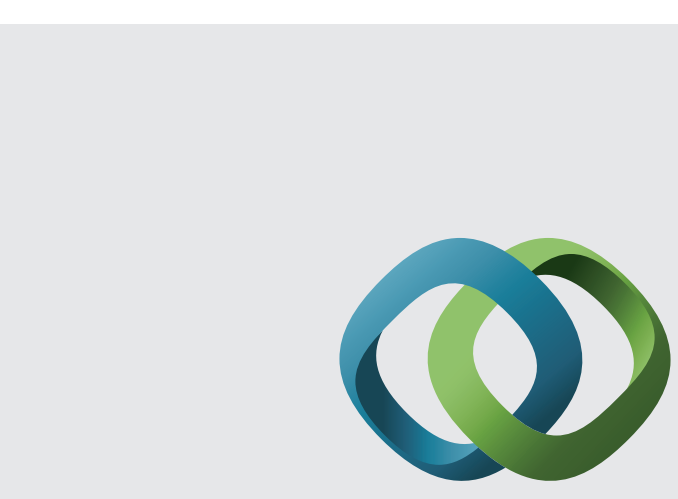

\section{Hindawi}

Submit your manuscripts at

http://www.hindawi.com
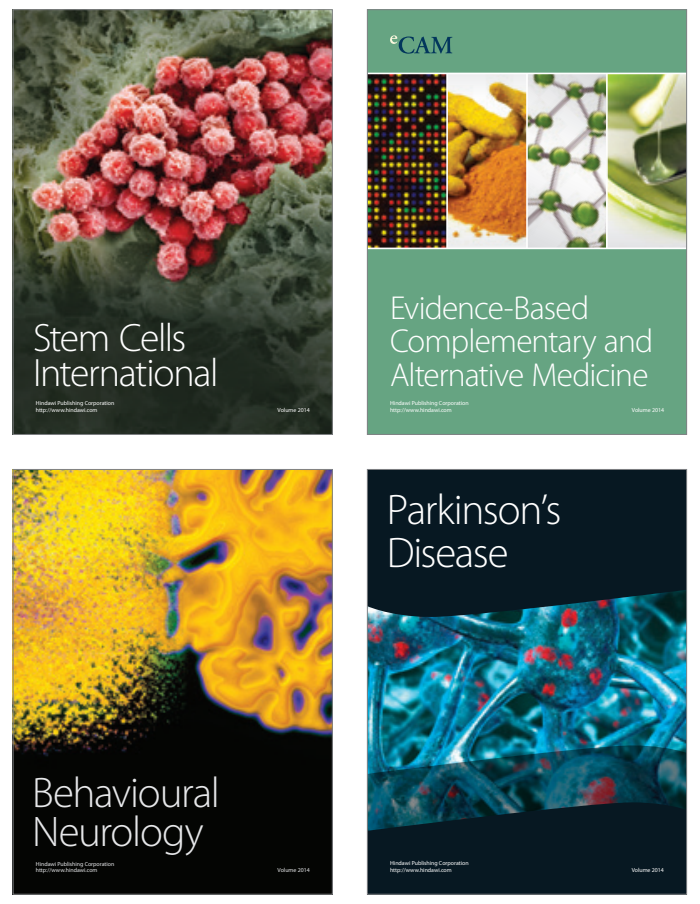
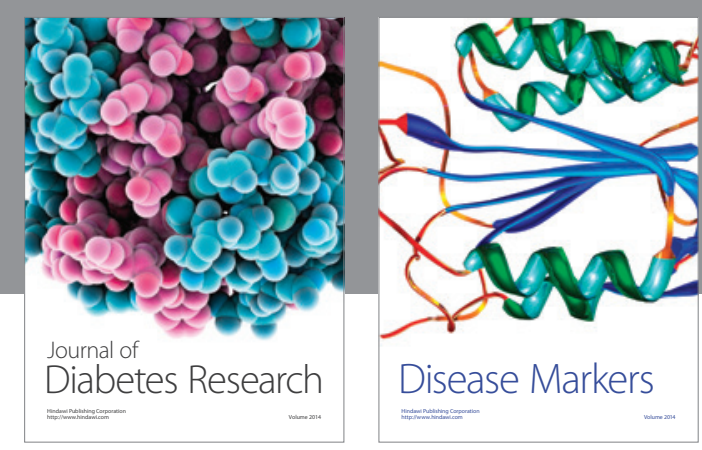

Disease Markers
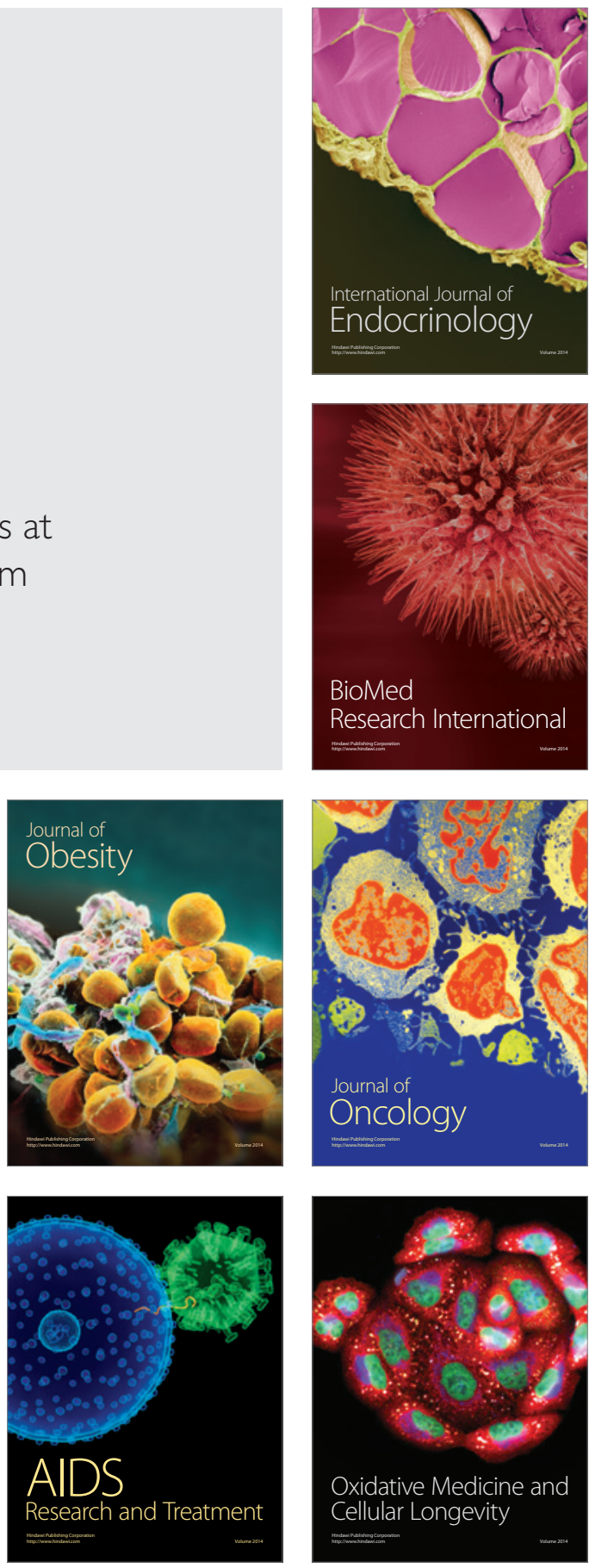